Hydroécol. Appl. (1997) Tome 9 Vol. 1-2, pp. 195-211

\title{
Les radeaux de végétation de la retenue de Petit-Saut (Guyane)
}

\section{Floating vegetation in Petit-Saut reservoir (French Guiana)}

\author{
Gérard Blake ${ }^{(1)}$ \\ (1) Laboratoire de Biologie et Biochimie Appliquées ESIGEC. Université de Savoie \\ 73376 Le Bourget-du-Lac
}

\begin{abstract}
Résumé. - Les retenues artificielles de milieux tropicaux sont la proie, les premières années de leur existence, d'un développement excessif de plantes aquatiques flottantes qui peuvent créer des nuisances et renforcer certains problèmes d'exploitation. La retenue de Petit-Saut présentait un risque élevé d'apparition de jacinthes d'eau (Eichhornia crassipes) car cette plante est reprèsentée sur le littoral guyanais. Le suivi de la végétation effectué dès la mise en eau n'a mis en évidence que l'apparition de radeaux portant essentiellement des hélophytes (Ptéridophytes, Poacés et Cypéracés) et hydrophytes. L'évolution de la composition floristique des radeaux montre une dominante d'espèces colonisatrices typiques des savanes de Guyane.

II est remarquable de noter l' abscence de macrophytes flottantes et la prépondérance d'hélophytes nécessitant un support de fixation.

Ces végétaux montrent une grande capacité de croissance que compensent en grande partie les processus de décomposition mesurés par différentes voies au sein des radeaux (pertes de biomasse de différents supports végétaux présentant un $k$ moyen de 0,0075 jour $^{-1}$ ). Les mesures effectuées in situ mettent en évidence des cinétiques de décomposition traduisant la dominante cellulosique du matériel végétal. L'excès de matière en décomposition des radeaux, placée au sein de la couche oxygénée de surface de la retenue se traduit par des caractéristiques élevées de réduction chimique auxquelles les hélophytes peuvent résister par des adaptations physiologiques propres à ces plantes de marais.
\end{abstract}

Mots-clés. - Macrophytes, végétation aquatique, retenue artificielle, radeaux, décomposition.

Abstract. - Artificial tropical resenoirs are often covered in their first years by macrophytes that can disrupt hydroelectric plant operations. Petit-Saut reservoir presented a high risk of development of water hyacinths (Eichhornia crassipes), found along the coast of French Guiana. Monitoring of aquatic plants, requested by Electricité de France, pointed up only the appearance of floating-mats covered by helophyte species (Pterido- 
phytes, Poacea and Cyperacea) and a few hydrophytes. The evolution of the composition of the flora in the mats reveals the predominance of colonizing species typical of the savanna of French Guiana.

It is important to note the absence on these floating-mats of floating macrophytes and the preponderance of helophytes which require support in order to settle.

These species show a great capacity for growth, offset to a large degree by the processes of decomposition measured in various ways on the mats (losses of biomass from various types of plant support amounting to an average $\mathrm{k}$ of $\left.0.0075 \mathrm{day}^{-1}\right)$. Field measurements point up kinetics of decomposition indicative of the predominance of cellulose in the plants. Thanks to their physiological capacities for adaptation, these plants can withstand the reductive chemical characteristics of the substratum.

Key-words. - Macrophytes, aquatic vegetation, reservoir, floating-mats, decomposition.

\section{INTRODUCTION}

II est connu que les retenues artificielles situées en zone tropicale sont très souvent envahies au cours de leurs premières années d'existence par des plantes flottantes telles que Eichhornia crassipes et Salvinia molesta (Shelpe, 1961; Mitchell, 1972). La retenue de Petit-Saut présentait ce risque, en raison de sa situation et de la présence de plusieurs especces de macrophytes aquatiques en Guyane. L'objectif de notre mission est donc d'aborder l'évolution de toutes les formes de végétation aquatique supérieure sur cette retenue dès sa création. Un pré-inventaire floristique préliminaire est présenté ainsi que les relations entre la présence de la végétation présente sur des radeaux et la décomposition de la matière végétale issue de ces plantes.

Les radeaux de végétation flottante sont relativement fréquents en milieu tropical (Reid, 1952; Bond et Roberts, 1978) et parfois présents en zone tempérée (Haraguchi, 1991). Leur origine traduit soit, de fortes productivités végétales de macrophytes flottants comme Eichhornia crassipes, Salvinia spp., Pistia stratiotes... etc. non compensées par un taux de décomposition en raison de l'installation d'une couche anaérobie au-dessous, soit des conditions hydrauliques particulières qui favorisent le détachement des végétaux de leur support d'origine (Blake, 1985).

Dans le cas de la retenue artificielle de Petit-Saut (Guyane), les radeaux de végétation sont apparus dans la région centrale (fig. 1), en novembre 1994, lorsque le niveau des eaux a atteint $31 \mathrm{~m}$, au niveau du barrage et, que les valeurs de conductivité et de teneurs en nitrates étaient élevées.

La présence de troncs d'arbres flottant issus d'anciennes coupes effectuées pour l'orpaillage explique le développement d'une quarantaine de petits radeaux dont certains sont uniquement constitués d'un tronc surmonté de touffes de Cypéracées 


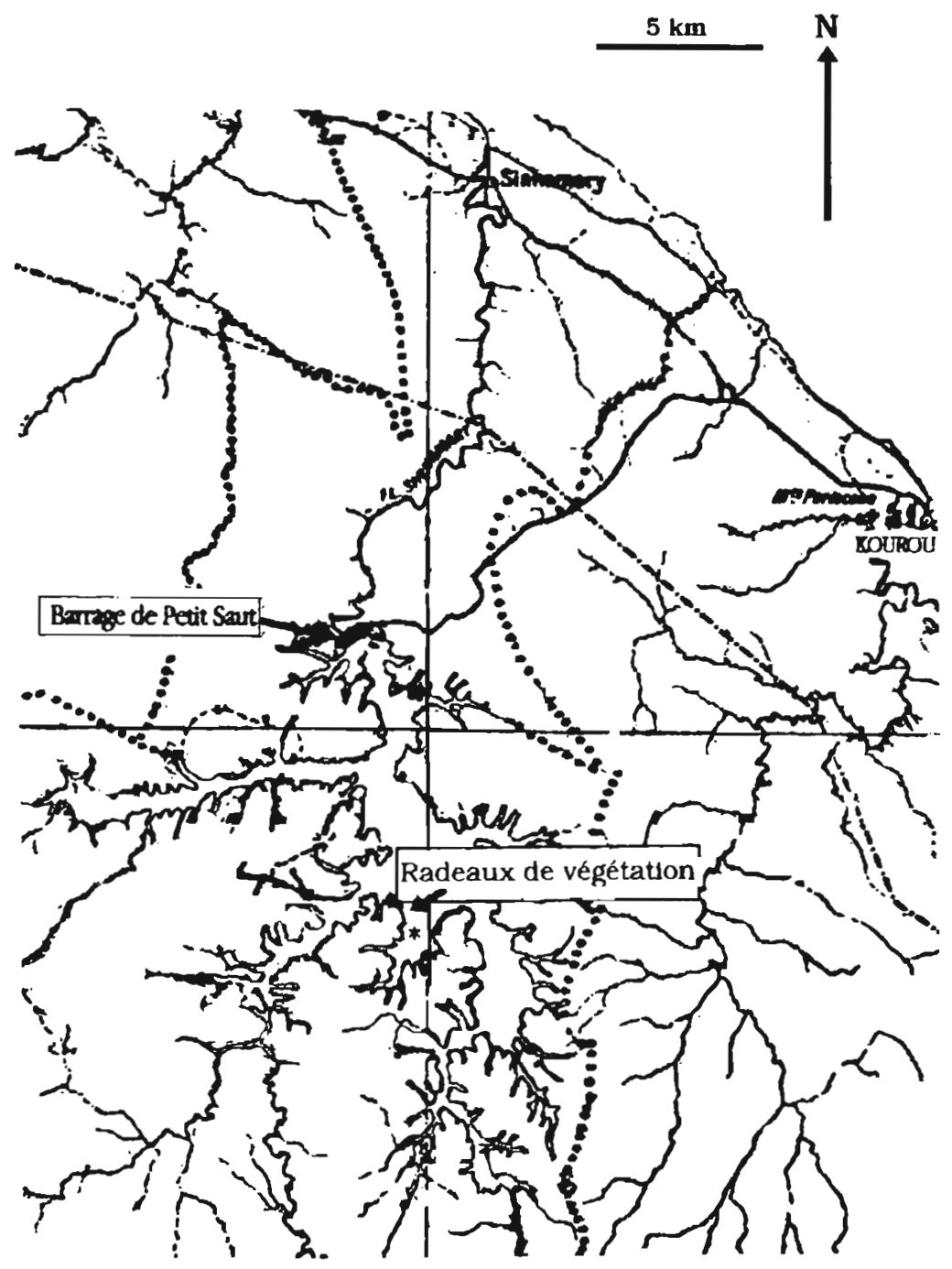

Fig. 1. - Situation des radeaux de végétation de la retenue de Petit-Saut.

Fig. 1. - Localisation of floating mats in Petit-Saut Reservoir.

(fig. 2) et d'autres constitués d'une masse plus conséquente d'hélophytes portée par ces troncs (fig. 3). II importait donc d'effectuer un suivi de ces radeaux afin de comprendre leur développement et les intéractions de ces radeaux avec le milieu de la retenue. 


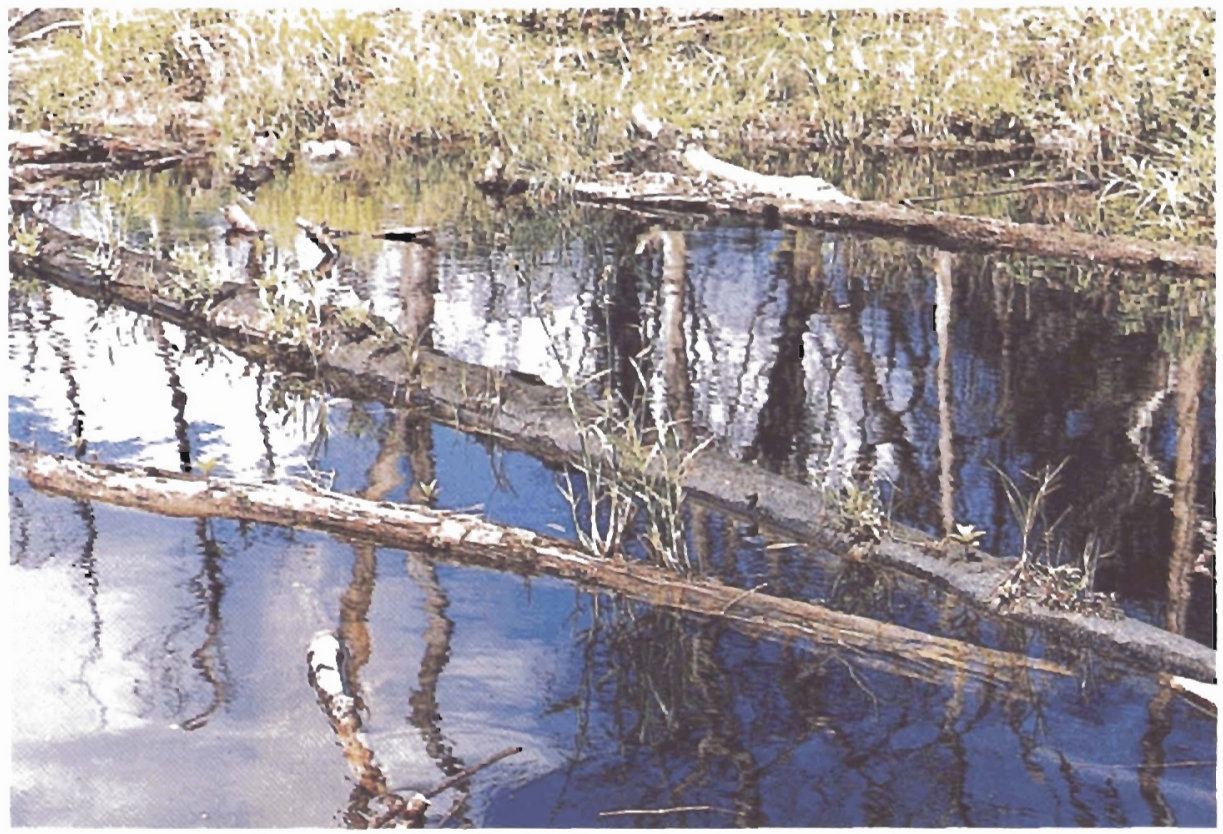

Fig. 2. - Troncs d'arbres au stade préliminaire de colonisation.

Fig. 2. - Preliminary colonisation on trees.

\section{MÉTHODOLOGIES}

\section{1 Étude de la végétation des radeaux}

Au cours de trois campagnes de relevés et de mesures, en 1995 et 1996, la composition floristique des radeaux a été relevée à l'aide de quadrats de $50 \mathrm{~cm}$ de coté en trois zones aléatoires pour les zones centrales des deux plus grands radeaux. Cette surface a été sélectionnée afin de ne pas déséquilibrer la structure des radeaux prospectés. De plus, des transects de relevés ont été réalisés perpendiculairement sur les bords des radeaux. La liste des espèces a été dressée en relevant tous les échantillons de plantes des quadrats. De plus, des mesures de biomasse ont été effectuées par prélèvement direct de la biomasse épigée des radeaux.

La liste des espèces dressée grâce à l'aide de G. Cremers de l'ORSTOM de Cayenne, figure en annexe 1.

\section{2 Étude des caractéristiques physico-chimiques des eaux interstitielles et des abords des radeaux}

Les mesures ont été effectuées à l'aide d'appareils portables Schott ${ }^{\text {(2) }}$ ( $\mathrm{pHmètre,} \mathrm{Oxymètre,} \mathrm{conductimètre} \mathrm{et}$ RedOxymètre). En avril 1996, un 


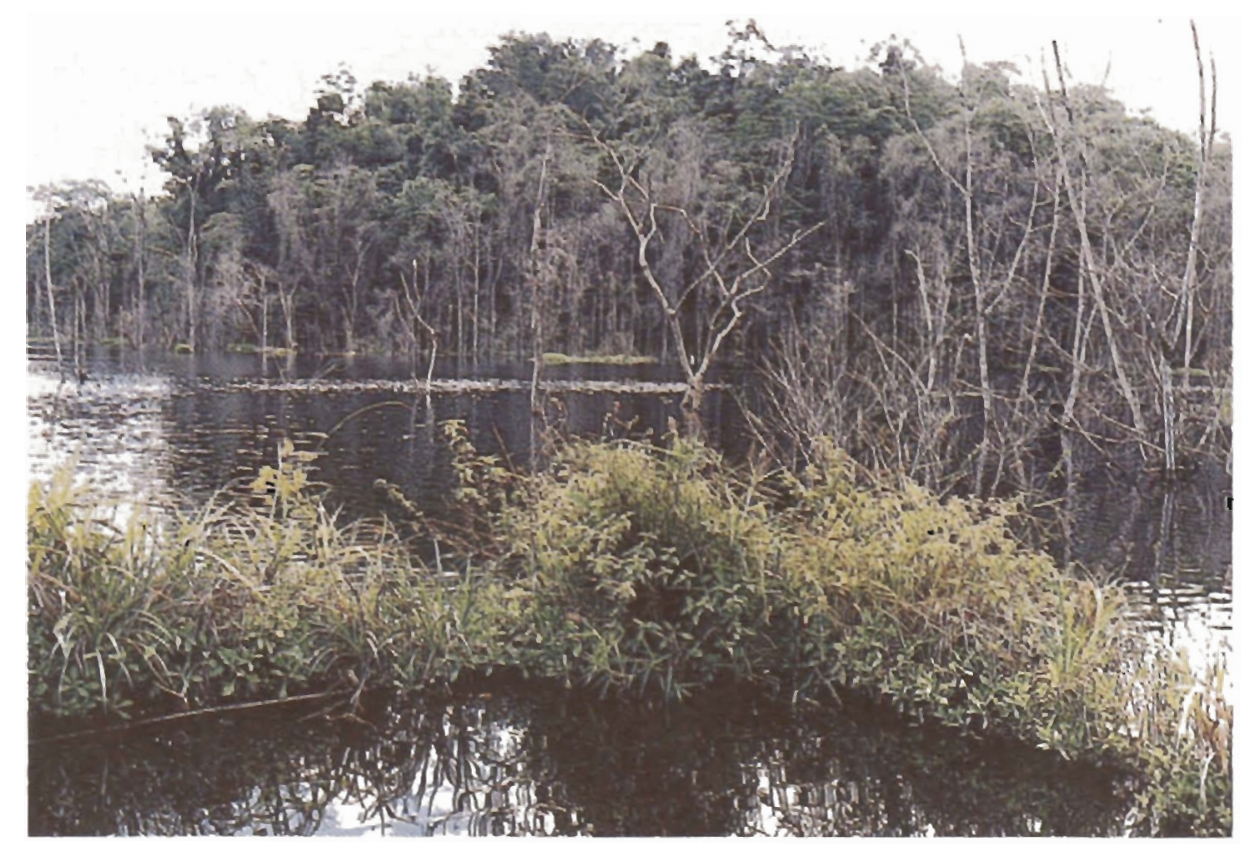

Fig. 3. - Stade avancé de colonisation de troncs d'arbre; formation de radeaux.

Fig. 3. - Advanced step for colonisation of trees. Mat early building.

spectromètre UV Secomam ${ }^{\circledast}$ portable a été utilisé pour mesurer in situ les teneurs en nitrates, le COT et les MES en différents points des radeaux et à proximité de ceux-ci.

\section{3 Étude de la décomposition de la matière végétale}

La décomposition de la matière végétale est le problème majeur lié à l'immersion de la biomasse de la forêt primaire (Pereira, 1993; Richard et al., 1996). Elle a été suivie sur place par différents types de matières d'origine végétale :

1) des feuilles d'arbres ( 6 feuilles de Panacoco (Swartzia tomentosa), 4 feuilles de Ouakapou (Annona sp.) (et
2 folioles de Cecropia sp. par sac de litière). Ces sacs, de deux mm de maille, ont été disposés en différents points de la retenue et à différentes hauteurs d'immersion et plus particulièrement pour certains, dans les radeaux de végétation; ces sacs sont régulièrement retirés puis leur contenu est lavé et pesé ;

2) des fragments de litière de radeaux placés dans de petites cages percées en acier inoxydable sont disposés au sein du substrat de ces mêmes radeaux; ces fragements sont retirés après plusieurs jours d'immersion au sein du radeau puis pesés;

3) des fragments de papier de cellulose sont fixés dans le substrat des radeaux depuis la surface jusqu'à une 
profondeur de $40 \mathrm{~cm}$. Ce substrat est destiné à détecter plus spécifiquement la vitesse de décomposition de la cellulose.

Tous ces échantillons de composés organiques sont pesés initialement et après leur temps d'immersion dans le milieu afin de connaitre le taux de dégradation dans les conditions subies in situ.

En complément de ces techniques de mesure par perte de poids, des mesures d'activité enzymatique (cellobiohydrolases) ont été effectuées par l'utilisation du p-nitrophényl- $\beta$-D-cellobiose à $5 \mathrm{mM}$ qui développe une couleur jaune mesurée à $410 \mathrm{~nm}$ (paranitrophénol) après une incubation de 2 heures in situ. La détection de cette activité enzymatique a été réalisée sur le terrain à l'aide d'un spectromètre $\mathrm{Hach}^{(\circledast)}$ afin d'obtenir une mesure correspondant aux enzymes présentes dans le milieu.

Le substrat des radeaux a été analysé par les méthodes habituelles retenues pour les sédiments d'eau douce (Méthode Anne-Afnor 31.109pour le carbone, Azote Kjeldahl - Afnor 31.111, Phosphore assimilable par méthode Truog). Quatre échantillons ont été recueillis dans les deux radeaux de plus grande surface.

\section{RÉSULTATS}

\section{1 Évolution des radeaux depuis la mise en eau de la retenue}

Les grands radeaux de végétation décrits dès 1994 sont encore en place, apres un an et demi, même si leur situation a quelque peu évolué (repères GIS légèrement modifiés de moins de cent mètres). Une restructuration de certains radeaux a eu lieu par regroupement de radeaux de plus petite taille.

En revanche, on a noté à l'est de ces grands radeaux, en zone abritée, la dégénérescence de certains radeaux de petite taille par une forte accélération du processus de décomposition du substrat constituant le support du radeau. Par cette décomposition du substrat, la portance du radeau diminue fortement et les plantes subissent alors une diminution de productivité, ce qui entraîne sa régression par manque d'apport en matière organique endogène; l'ensemble régresse.

La composition floristique des grands radeaux reste globalement identique à celle relevée en juillet 1995, elle est légèrement modifiée par une augmentation en pourcentage des Poacees qui semblent profiter de l'élévation du niveau émergé des radeaux :

Tableau I. - Répartition en biomasse (M.S.) des principaux taxons (moyenne de trois quadrats de $1 \mathrm{~m}^{2}$ de radeau).

\begin{tabular}{|c|c|c|}
\hline ' & $\begin{array}{c}\text { masse } \\
(g)\end{array}$ & $\begin{array}{c}\text { pour- } \\
\text { cen- } \\
\text { tages }\end{array}$ \\
\hline$-\longrightarrow$ & - & - \\
\hline Fougères & 270 & 60 \\
\hline Ludwigia sp. (Onagracées) & 71 & 17 \\
\hline Sabicea sp. (Rubiacées) & 48 & 12 \\
\hline Poacées & 32 & 7 \\
\hline Fuirena (Cyperacées) & 17 & 3 \\
\hline Desmodium (Papilionacées) & 2 & 0.5 \\
\hline TOTAL & 440 & 100 \\
\hline
\end{tabular}


On note que suivant un transect (fig. 4), la composition reste très voisine depuis deux années.

L'équilibre entre la composition floristique et le niveau d'eau dans le radeau semble conditionner le développement des macrophytes. L'évolution de la composition floristique des radeaux est liée au processus de sédimentation de la matière végétale produite, c'est-à-dire à l'équilibre entre le processus d'apport de matière par la production primaire et la capacité de décomposition due aux organismes hétérotrophes et aux inverté- brés. Sur les radeaux de grande taille, la croissance d'espèces ligneuses (surtout épiphytes) est vite limitée par la faible portance du support. Des arbustes (Onagracées, Amaranthacées...) profitent de l'ombrage et du support des grandes familles herbacées pour coloniser les espaces libres des radeaux, alors que les Ptéridaphytes prolifèrent dans les emplacements dégagés (Thelypteris et Pityrogramma) ou riches en humus (Blechnum sp.). La plupart des espèces présentes sont, en fait, des plantes exigeantes en lumière, colo-
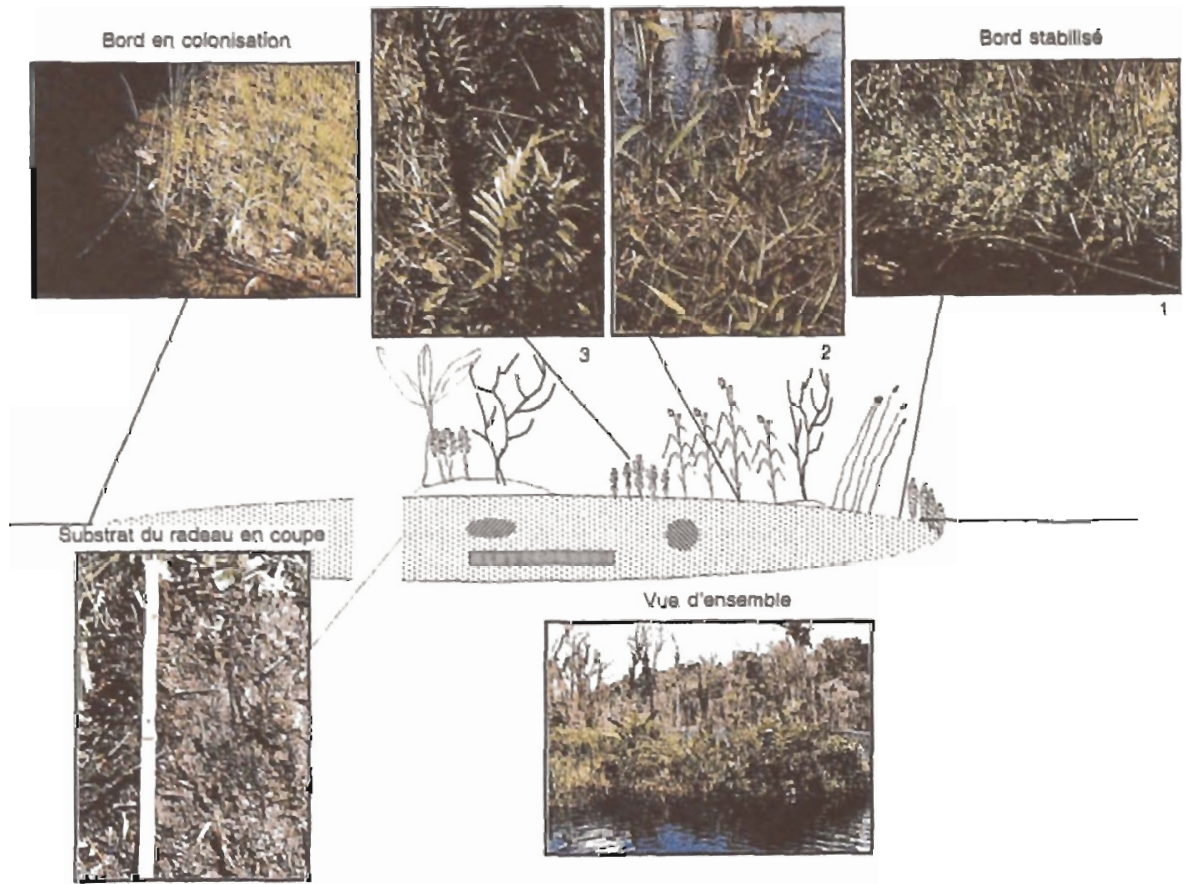

Fig. 4. - Composition simplifiée de la végétation d'un radeau.

Fig. 4. - Simplified plant composition of floating mat (global view, stabilized or extending edges). 
nisatrices et souvent présentes dans des terrains récemment remaniés (Cremers G. com. pers.).

\subsection{Présence d'organismes animaux sur les radeaux de végétation}

La présence des radeaux végétaux sur la retenue permet la présence de quelques espèces animales, liées pour la plupart aux végétaux par la nourriture.

Ainsi des Odonates (Caloptérydés, Coenagrionidés et Libellulidés), des fourmis (Dacetones amigerum), des chenilles de Saturnidés, des Diptères (Muscidés, Chironomidés, Limonides) des iules, des arachnides profitent de ce support et de la litière accumulée en surface. Il est difficile, pour l'instant de faire la part des Invertébrés ayant colonisé provisoirement ou définitivement ce type de milieu.

La couche organique du support des radeaux présente sur ses bords une couche en décomposition active, recouverte d'un biofilm dense d'organismes périphytiques dont la composition est voisine de celle que l'on relève sur les amas de végétaux terrestres en décomposition présents dans la retenue.

Dans ce périphyton dominé par une couche algale dense marquée par des algues vertes filamenteuses (Spirogyra sp, Mougeotia sp. et de nombreuses diatomées pennées) et des protozoaires typiques des eaux chargées en matière organique en décomposition, (stentors, vorticelles,
Stylonichia), les populations zooplanctoniques sont surtout représentées par des Cladocères de petite taille (Bosminidées, Sididées, des ostracodes et des Calanoïdes); si la comparaison de la composition zooplanctonique est difficile à faire entre la zone pélagique et ce périphyton, il semble que le périphyton soit relativement plus riche en ostracodes et petits cladocères que les eaux entourant les radeaux. II a été montré dans ces régions tropicales que les radeaux étaient des milieux qui augmentaient la biodiversité des écosystèmes dans lesquels ils se développaient (Junk, 1973).

\section{3 Évolution floristique des radeaux}

La composition floristique habituelle des radeaux se rapproche en fait de celle que l'on trouve dans certaines savanes humides du littoral guyanais (Champeau et al., 1991) et l'évolution légère de cette composition décelée sur une année et demi est représentée par une flèche sur la figure 5. La flèche symbolise sur cette figure, l'évolution des dominantes des compositions floristiques des radeaux au cours de notre suivi (les positions des axes et des espèces signalées par les auteurs cités ont été reprises simplement sans nouveau calcul, compte tenu du faible nombre de donnés dans notre situation). Cette évolution ne semble pas s'orienter pour l'instant vers l'apparition de macrophytes flottantes (Eichhornia cras- 


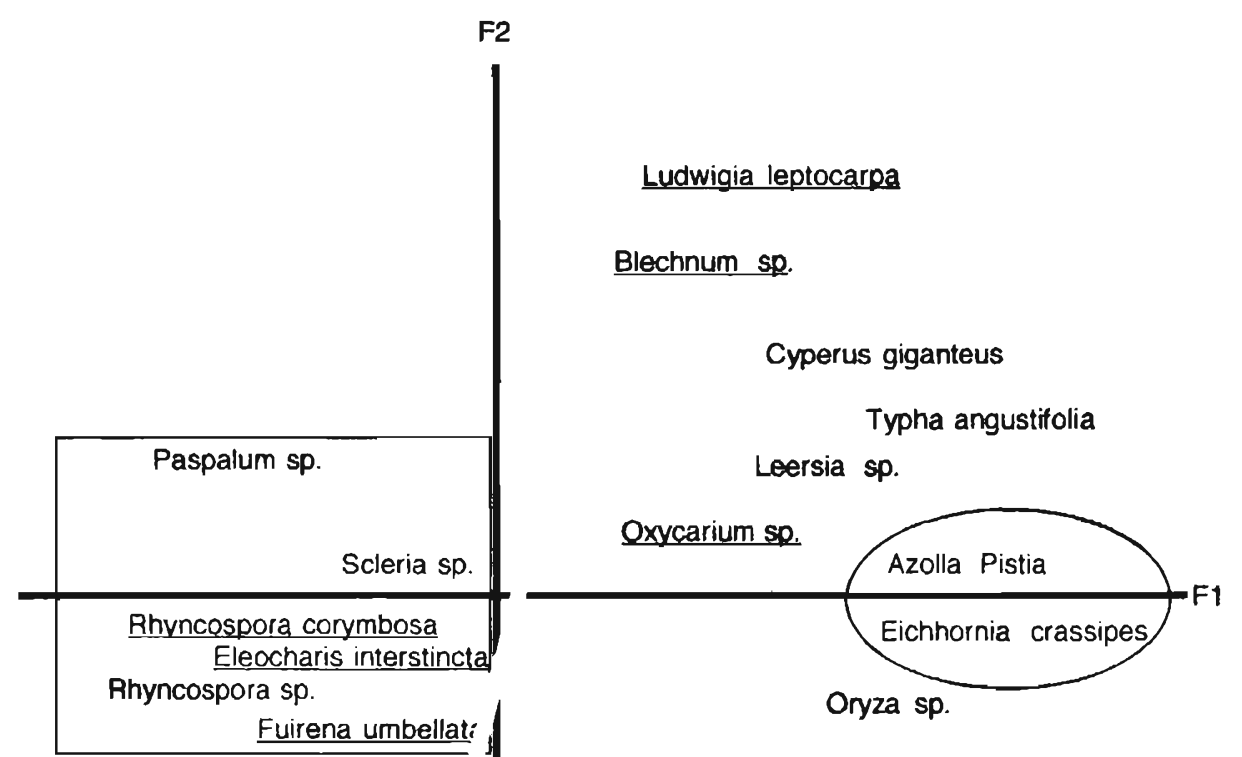

Tonina fluviatilis:

Cabomba aquatica

Fig. 5. - Evolution de la composition floristique des radeaux (flêche). Siłuation par rapport à la distribution des especes décrites par Champeau et al. (1991). Les espèces typiques des radeaux sont en souligné. Les espèces flottantes sont entourées d'un cercle (à droite); les espèces de savanne, à gauche dans un rectangle. Les axes F1 et F2 correspondent aux axes de l'étudeanalyse factorielle citée.

Fig. 5. - Floristic composition of floating mats from the beginning to the present state (arrow). This composition is presented, following the distribution of species of Champeau et al. study (1991). Floating spp. are circled on the right and savanna spp. in rectangle on the left part of figure. F1 et F2 are axes of FCA of the published study.

sipes, Salvinia sp. ou Pistia stratiotes) comme cela a pu être décrit dans de nombreuses retenues artificielles tropicales voisines d'Amérique du Sud, telle que Tucurui, au Brésil. Les stations de Guyane, dans lesquelles ont été signalées par Champeau et al. (1991) ces espèces, et revisitées par nous-mêmes, sont toutes situées sur le littoral et marquées par des eaux souvent enrichies en éléments nutritifs minéraux liés aux activités anthropiques (élevages, cultures, rejets domestiques concentrés...); les caractéristiques de ces eaux figurent en annexe 2 et sont illustrées par l'annexe 3. 
Le risque d'apparition d'espèces flottantes, même s'il est relativement plus faible qu'au cours des deux premières années de mise en eau, reste toujours présent et surtout de plus en plus lié à toute évolution de la qualité des eaux (apports nutritifs par augmentation des niveaux de fréquentation par l'homme, accroissement de la vitesse de minéralisation de la charge de matière organique présente) et à une contamination par importation de graines ou de turions de plantes (due également par l'augmentation de la fréquentation de la circulation de bateaux ou par phorésie).

II a été montré, également (Sutton, 1989) que la présence d'Eleocharis interstincta pouvait limiter la croissance de macrophytes à feuilles flottantes comme Lemna paucicosta par l'émission de substances à rôle allélopathique.

\subsection{Composition chimique du support des radeaux et incidences de la présence de la matière organique sur l'eau de surface de la retenue}

Le substrat des radeaux est de composition relativement hétérogène de par sa formation (présence de troncs en décomposition, de branches, de feuilles et de macrophytes en voie de décomposition).

Le tableau II présente la composition moyenne de la couche tourbeuse qui constitue le remplissage du corps des radeaux:
Tableau II. - Composition moyenne du support des radeaux (2 radeaux de grande surface : 4 échantillons par radeaux).

$\begin{array}{lr}\text { Carbone organique en } \% & 48,3(+/-5,5) \text { : } \\ \text { Azote total en \% } & 2,2(+/-0.4) \\ \text { Phosphore assimilable en \% } & 2.2(+/-0,2) \\ \text { Calcium en } \mathrm{mg} / \mathrm{kg} \mathrm{MS}: & 7144(+/-50) \text { : } \\ \text { Potassium en } \mathrm{mg} / \mathrm{kg} \text { MS : } & 416(+/-15) \text { : }\end{array}$

Cette composition montre que les éléments minéraux (notamment l'azote en raison du processus de dénitrification) peuvent être relativement limitants pour la croissance végétale épigée et que cette croissance ne peut s'expliquer que par un remaniement rapide de la biomasse qui permet une bonne utilisation des éléments stockés. Le $\mathrm{C} / \mathrm{N}$ de 21,6 traduit le fait que la matière végétale est sous un état encore peu minéralisé avec une origine de végétal supérieur dominante, dans la région centrale des radeaux.

\subsection{Décomposition de la matière organique}

Il a été souvent montré que le processus de décomposition était sous la dépendance de plusieurs paramètres du milieu tels que le potentiel d'oxydoréduction, la présence de composés azotés minéraux nécessaires aux animaux, et bien-sûr la densité des populations de micro-organismes hétérotrophes. Dans le cas des radeaux de la retenue, situés dans la couche de surface, au-dessus de l'oxycline, une part importante de la décompo- 
sition se fait en aérobiose. Par contre, dès une profondeur de $5 \mathrm{~cm}$ à l'intérieur de la tourbe, le potentiel d'oxydoréduction chute et atteint des valeurs qui témoignent que les voies de décomposition anaérobies sont prédominantes (fig. 6). En fait, à l'échelle de l'épaisseur du radeau, la situation est plus complexe car la plupart des hélophytes (Armstrong, 1989) et en particulier les Cyperacées (Li et Jones, 1995) ont la propriété d'apporter à proximité de leurs racines une certaine quantité d'oxygène par leur lacune aérifère et d'évacuer des excès de gaz issus des activités de décomposition bactérienne comme le méthane et le dioxyde de carbone. Ce rôle des hélophytes permet l'association de populations hétérotrophes aérobies et anaérobies au voisinage de la rhizosphère, ce qui contribue à obtenir un taux élevé de décomposition en dépit de conditions potentiellement défavorables.

La figure 7 met en évidence les cinétiques de décomposition des différents composés organiques d'origine végétale. Les courbes de décomposition des composés complexes (feuilles d'arbres) présentent une cinétique classique marquée par une première phase de lessivage des hydrosolubles de 0 à 12 jours (avec

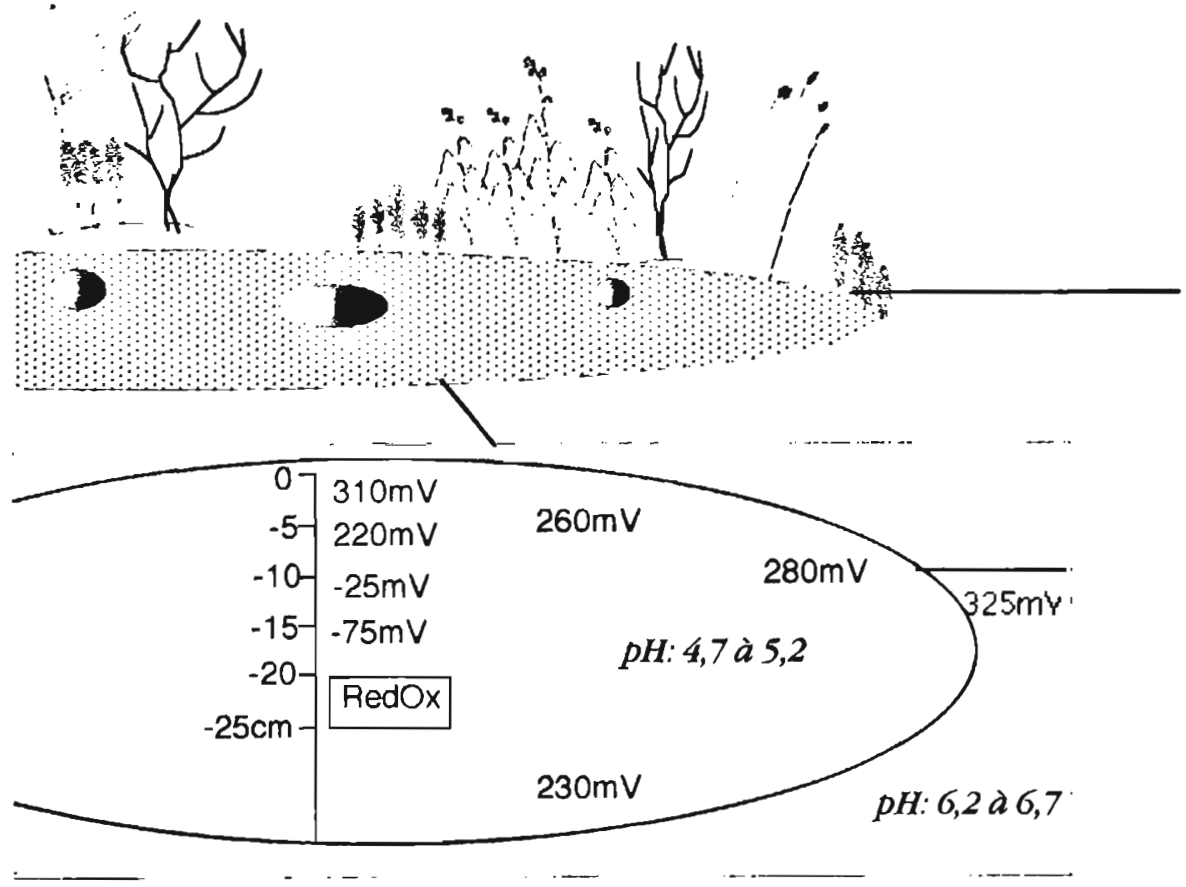

Fig. 6. - Distribution du RedOx et du pH dans le substrat des radeaux.

Fig. 6. - RedOx and $\mathrm{pH}$ measurements in different parts of the mat. 
Pourcentage de perte de poids

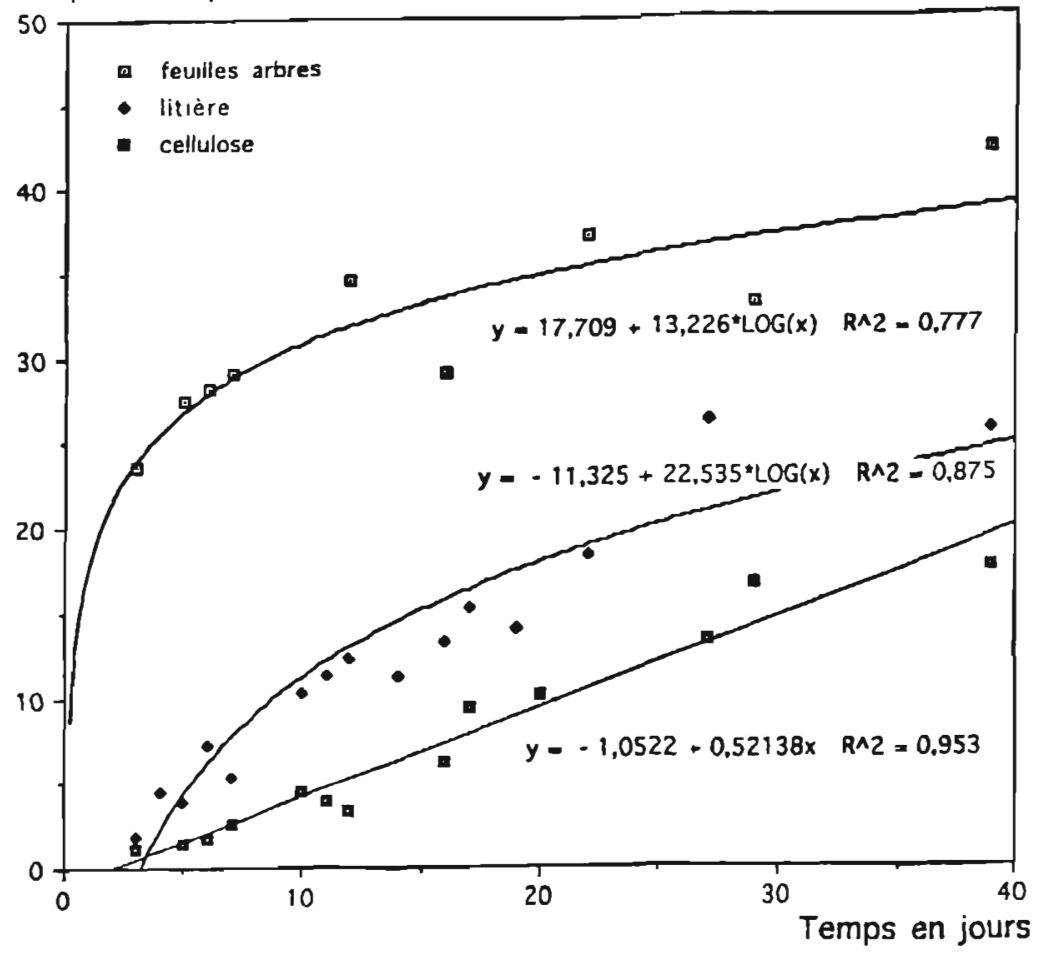

Fig. 7. - Cinétique de décomposition de la matière végétale dans la retenue de Petit-Saut.

Fig. 7. - Kinetics of degradation of plant material in Petit-Saut Reservoir.

un $\mathrm{k}$ de 0,055 jour $^{-1}$ ) puis un ralentissement du taux de dégradation par la suite ( $k$ de 0,016 jour ${ }^{1}$ ), correspondant à la phase de dégradation des composés de structure du végétal; pour la litiere de radeau, la forme générale du processus est identique (coefficient de corrélation $R^{2}=0,875$ ) mais le taux $k$ moyen est de 0,0075 jour $^{-1}$. La cellulolyse étudiée par perte de poids des carrés de cellulose montre une vitesse pratiquement constante du fait que le processus est engagé depuis plus d'un an dans la retenue et que la densité des organismes cellulolytiques est élevée; dans ces conditions, la valeur moyenne du taux est de: $k=0,0083 j^{-1}$.

On peut estimer à partir de ces cinétiques, les temps de disparition de $95 \%$ de chaque biomasse ( $195 \%$ ); dans le cas de cinétiques logarithmiques, les valeurs sont les suivantes:

t95\% $=190$ à 200 jours pour les feuilles d'arbres, $195 \%=400$ jours pour la litière des radeaux, 
$195 \%=600$ jours pour la cellulose pure, en aérobie,

$195 \%=800$ jours pour la cellulose pure, en anaérobie.

Les valeurs théoriques du taux de décomposition $(k)$ relevées dans la littérature, pour des situations comparables sont du même ordre de grandeur $(0,0071$ à 0,0074 pour l'espèce Paspalum sp., Howard-Williams et Junk, 1976 et 1977).

Dans le cas des radeaux, il est vrai que la présence de supports et de surface de fixation supplémentaire pour les micro-organismes hétérotrophes, permet de développer, au moins sur les bords et la surface des radeaux, une activité de dégradation plus importante qu'en pleine eau, à proximité de ces mêmes radeaux. Cette plus forte activité cellulolytique se vérifie par les mesures effectuées in situ (tableau III).

Ces valeurs mettent en évidence le rôle que jouent les radeaux dans la participation à l'activité de décomposition de la matière végétale présente dans la couche oxygénée de la retenue. La figure 7 met en évidence la légère supériorité de la vitesse de dégradation de la matière végétale dans les couches superficielles des radeaux, par rapport à leur environnement.

Tableau III. - Activités enzymatiques cellulolytiques mesurées dans la retenue en avril 1996.

Dans l'eau interstitielle des radeaux : 2,3 à 2,8 moles de substrat consommées. $h^{-1}$.

Dans l'eau voisine (couche de surface) : 1,6 à 2,4 moles de substrat consommées. $h^{-1}$.

\section{DISCUSSION}

L'absence de macrophytes à feuilles flottantes de type jacinthe est pour l'instant une situation qui distingue l'évolution de la retenue de PetitSaut de celle de nombreuses retenues voisines du Brésil, du Surinam ou même d'Afrique. La prépondérence des plantes enracinées, qui avait été prévue, il faut le souligner, dans l'étude d'impact, pose toute de même un certain nombre de questions sur l'origine de l'absence de développement des plantes flottantes:

- absence d'espèces à l'origine, lors de la montée des eaux (raison principale, à notre avis),

- effet des vagues,

- présence d'une forte prédation de consommation.

Les expérimentations effectuées par des tests de croissance de lentilles d'eau (Lemna minor, Blake, 1995) montrent une disponibilité d'éléments nutritifs et une absence d'élements phytotoxiques qui laissent à penser que tout développement de plantes flottantes est encore possible sur la retenue.

La présence de radeaux portant des hélophytes, des Ptéridophytes, quelques hydrophytes, des Bryophytes et des espèces ligneuses met en évidence une forte capacité de production végétale si des conditions particulières sont favorables (troncs flottants, zones abritées des vents, proximité des sédiments...). Ces radeaux sont en soit de véritables microcosmes dans lesquels, les équi- 
libres entre production et décomposition de matiere organique sont fragiles (Rai et Munshi, 1979). Nos résultats montrent des gradients d'oxydoréduction élevés à l'intérieur du support de ces radeaux, qui dans cette situation à température relativement élevée tout au long de l'année conduit à la sélection de souches de microorganismes hétérotrophes sans doute très adaptées.

L'évolution de la composition floristique de ces radeaux est en fait la résultante de cet équilibre entre production et décomposition de matière puisque les ligneux ne peuvent s'installer qu'au dessus d'un certain niveau émergé. Ces radeaux constituent donc de bons témoins des équilibres écologiques de la couche de surface de la retenue.

\section{CONCLUSION}

La retenue de Petit-Saut ne présente pas, un an et demi à deux ans après son remplissage complet, un développement de macrophytes à feuilles flottantes comme cela a été observé dans de nombreuses retenues tropicales. Cette situation peut s'expliquer partiellement par une situation écologique privilégiée et surtout par une absence d'activités humaines génératrices d'apports nutritifs excessifs. La présence de radeaux flottants composés d'hélophytes Cypéracées et de Ptéridophytes est une particularité de cette retenue. Leur composition évolue peu dans le temps et se rapproche de celle des savanes humides du littoral guyanais. Le support du radeau constitue essentiellement de bois, de feuilles, de macrophytes constitue un ensemble en décomposition aérobie et anaérobie suivant des gradients d'oxydoréduction de par le fait que cette masse de matiere organique se trouve dans la couche oxygénée de surface de la retenue. De plus, les hélophytes qui poussent sur ces radeaux sont capables d'apporter de l'oxygène au sein du radeau par leur activité photosynthétique; ce qui contribue à accélérer l'activité hétérotrophe.

Les taux de décomposition mesurés sur des feuilles d'arbre, de la litière et des fragments de cellulose montrent des valeurs voisines de celles relevées habituellement en zone tropicale et liées au substrat décomposé.

Ces mesures témoignent d'une forte activité cellulolytique amplifiée par la présence de supports de fixation pour les bactéries. II est certain que le développement des radeaux dans cette couche de surface se traduirait par une augmentation des vitesses de décomposition de la matière organique présente.

Le suivi de la composition floristique et faunistique des radeaux, des mécanismes de production et décomposition de la matière végétale peut permettre de comprendre dans l'avenir la tendance évolutive du cycle de la matière organique dans cet écosysteme actuellement fortement marqué par la dystrophie. 


\section{REMERCIEMENTS}

Ces travaux de recherches ont été réalisés dans le cadre du contrat EDF-Université de Savoie. ESIGEC $n^{\circ} \mathrm{CNEH}$. 7586-PE A 750.

\section{RÉFÉRENCES \\ BIBLIOGRAPHIQUES}

Armstrong W., 1989. Aeration in roots. Environmental stress in plants. In (J.H. Cherry ed.) : 197-206.

Blake G., 1985. Production et Décomposition des macrophytes aquatiques. Thèse d'Etat de I'Univ. de Grenoble: 135 p. + annexes.

Blake G., 1995. Etude des radeaux de végétation flottante de la retenue de $\mathrm{Pe}$ tit-Saut. Rapport d'étude EDF: $51 \mathrm{p}$.

Bond W.J., Roberts M.G., 1978. The coIonization of Cabora basa, Mosambique, a new man-made lake, by floating aquatic macrophytes. Hydrobiologia, 60 (3) : 243-259.

Champeau A. et al. 1991. Petit-Saut hydroelectric scheme: vegetal associations of stagnant waters in French Guyana. Hydroécol. Appl., 1: 111-124.

Haraguchi A., 1991. Effects of water-table oscillation on redox property of peat in a floating mat. Ecol., 79 : 1113-1121.

Howard-Williams C. et Junk W.J., 1976. The decomposition of aquatic macrophytes in the floating meadows of a central amazonian varzea lake. Biogeographica, 7 : 115-123.
Howard-Williams C. et Junk W.J., 1977. The chemical composition of central amazonian macrophytes with special reference to their role in the ecosystem. Arch Hydrobiol., 79 : 446-464.

Junk W.J., 1973. The aquatic fauna of the root zone of floating vegetation. Amazoniana, $4:$ 9-12.

$\mathrm{Li} \mathrm{M}$. and Jones M., 1995. $\mathrm{CO}_{2}$ and $\mathrm{O}_{2}$ transport in the aerenchyma of Cyperus papyrus L. Aquatic Botany, 52 : 93106.

Mitchell D.S., 1972. The Kariba weed: Salvinia molesta. Brit. Fern. Gazzett, $10(5)$ : 251-212.

Pereira A., 1993.- Décomposition de la matière organique. Rapport EDF/CERGRENE : $47 \mathrm{pp}$.

Rai D.N., Munshi J.D., 1979. The influence of thick floating vegetation (Water hyacinth: Eichhornia crassipes) on the physicochemical environment of a fresh water wetland. Hydrobiologia, 62 (1) : 65-69.

Reid G.K., 1952. Some considerations and problems in the ecology of floating islands. J. Fla. Acad. Sci., 15 (1) : 6366.

Richard S. et al., 1996. Evolution de la qualité physico-chimique de l'eau du Sinnamary depuis le début de la mise en eau de la retenue. Hydroécol. appl. $9(1 / 2)$ : pp. 57-83.

Shelpe E.A.C.L.E., 1961. The ecology of Salvinia auriculata and associated vegetation on Kariba Lake. J.S. Afr. Bot., 27 : 181-187.

Sutton D., 1989. Allelopathy studies. Aquaphyte, 9 (1): 3. 


\section{Annexe 1}

Composition floristique des radeaux étudiés.

Floristic composition of floating mats.

CYPÉRACÉES : - Cyperus odoratus Linnaeus.

- Fuirena umbellata Rottb.

ÉRIOCAULACÉES : - Tonina fluviatilis Aubl.

PTÉRIDOPHYTES : - Blechnum cerrulatum Linnaeus.

- Thelypteris interrupta Schimdel.

- Pityrogramma calomelanos Link.

- Ceratopteris sp. Linnaeus

CONVOLVULACÉE : Ipomea sp. (patata?) Linnaeus.

APOCYNACÉE : Mandevilla sp (subspicata?) Lindley.

ONAGRACÉE : Ludwigia leptocarpa (Nuttall) Hara

CLUSIACÉE : Clusia sp. Linnaeus.

PAPILIONACÉE : Desmodium sp. Línnaeus

AMARANTACÉE : Clellosia $s p$. Linnaeus.

ORCHIDACÉE : Habenaria longicauda Willdenow.

POACÉES : deux especes, Hymenachne sp. et une indéterminée.

\section{Annexe 2}

Caractéristiques physicochimiques des stations à macrophytes flottantes relevées en Guyane.

Physicochemical characteristics of floating macrophytes stations of French Guyana.

I. D'après Champeau et al. (1991):

$\begin{array}{lcccc} & \mathbf{p H} & \begin{array}{c}\text { Tempèrature } \\ \left({ }^{\circ} \mathrm{C}\right)\end{array} & \begin{array}{c}\text { Conductivité } \\ (\mu \mathrm{S} / \mathrm{cm})\end{array} & \begin{array}{c}\mathrm{O}_{2} \text { dissous } \\ \left(\mathrm{mg} . \mathrm{l}^{1}\right)\end{array} \\ \text { Eichhornia crassipes } & \mathbf{4 , 7 - 5 , 8} & 26,7-33 & 11-80 & 4,6-9,2 \\ \text { Salvinia auriculata } & 3,7-7,4 & 25-33 & 5-1260 & 3,5-9,2 \\ \text { Pistia stratiotes } & 4,6-7,8 & 25,2-33,5 & 5-2710 & 3,1-6,4 \\ \text { Ipomea aquatica } & \mathbf{5 , 1 - 5 , 8} & 26,7-30,3 & 12-60 & 4,6-6,2\end{array}$

II. D'après les relevés de Blake (1996):

$\begin{array}{lccccc} & \mathrm{pH} & \begin{array}{c}\text { Température } \\ \left({ }^{\circ} \mathrm{C}\right)\end{array} & \begin{array}{c}\text { Conductivité } \\ (\mu \mathrm{S} / \mathrm{cm})\end{array} & \begin{array}{c}\mathrm{O}_{2} \text { dissous } \\ \left(\mathrm{mg} . \mathrm{l}^{-1}\right)\end{array} & \begin{array}{c}\text { Phosphates } \\ \left(\mathrm{mg} . l^{-1}\right)\end{array} \\ \text { Eichhomia crassipes } & 4,9-6,6 & 28-29,6 & 52-217 & 4,3-6,6 & 0,060-0,820 \\ \text { Salvinia auriculata } & 4,2-6,0 & 27-30 & 38-1334 & 2,5-4,3 & 0,060-0.120 \\ \text { lpomea aquatica } & 4,8-5,3 & 28-32 & 25-95 & 4,5-7,2 & 0,015-0,048\end{array}$




\section{Annexe 3}

Stations à Eichhornia crassipes de Guyane.

Eichhornia crassipes stations of french Guyana.

\section{Présence d'Eichhornia crassipes dans des eaux char- gées en éléments nutritifs en Guyane: stations étudiées en avril 1996}

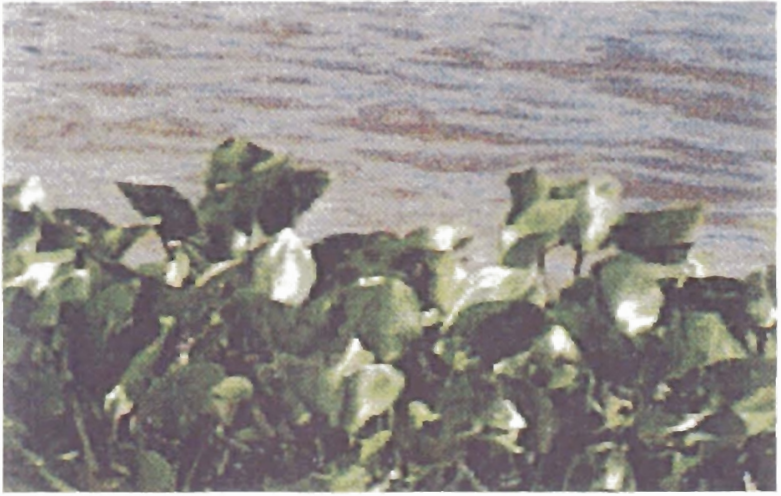

Radeaux de jacinthes sur la rivière Mana. Cette rivière transporte en période de hautes eaux des radeaux de jacinthes qui se déposent sur ses rives (photo prise à Mana). Les plantes échouées restent vivaces pendant plusieurs jours.

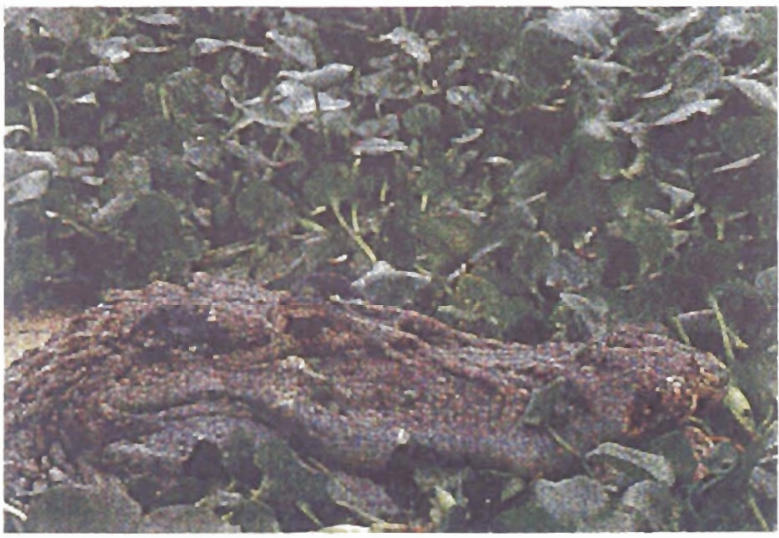

Présence de jacinthes dans une eau riche en éléments nutritifs: le bassin des caïmans de la réserve animalière de Montsinéry. Les macrophytes sont en pleine floraison dès le mois d'avril et leur productivité est élevée. Des jacinthes ont été placées dans d'autres bassins afin d'épurer les eaux.

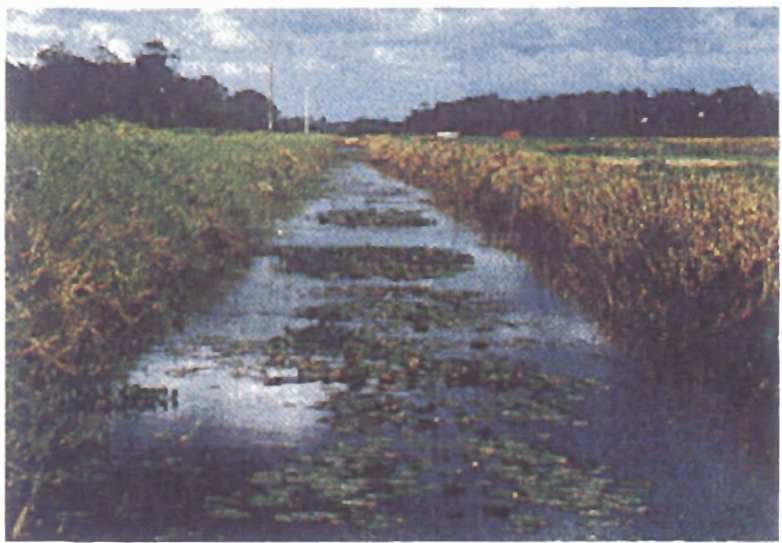

Autre exemple de situation à haut niveau trophique marquée par la présence d'E. crassipes : Ies canaux des rizières de Mana: ces canaux sont très chargés de résidus d'engrais (et pesticides) provenant des rizières proches. . On note également la présence de Nymphaea.

Ces présences signalées par Champeau et al. (1991) ne semblent pas avoir donné lieu à une expansión de l'espèce en zone littorale guyanaise. 